K. Funahashi

Nagoya Math. J.

Vol. 94 (1984), 89-104

\title{
NORMAL HOLOMORPHIC MAPPINGS AND CLASSICAL THEOREMS OF FUNCTION THEORY
}

\author{
KEN-ICHI FUNAHASHI
}

\section{§. Introduction}

In [7], O. Lehto and K.I. Virtanen introduced the concept of normal meromorphic functions in connection with the study of boundary behaviour of meromorphic functions of one complex variable.

In this paper, we generalize the theory of normal meromorphic functions to the case of holomorphic mappings into higher dimensional complex spaces in connection with the theory of hyperbolic manifolds and Nevanlinna theory.

The main concern of this paper is the generalizations of the big Picard theorem and Lindelöf's theorem which appear in the classical function theory.

\section{$\S 1$. Definition of normal holomorphic mappings}

In this section, we define the concept of normal holomorphic mappings similar to normal meromorphic functions (cf. [7]).

Let $M$ and $N$ be complex analytic spaces. We denote the set of holomorphic mappings from $M$ into $N$ by $\operatorname{Hol}(M, N)$. We say a subset $\mathscr{F}$ of $\operatorname{Hol}(M, N)$ to be a normal family if $\mathscr{F}$ is relatively compact in Hol $(M, N)$ in the sence of compact open topology.

Definition 1. Let $D$ be a homogeneous bounded domain in $C^{n}$ and $N$ be a complex analytic space. We say that a holomorphic mapping $f$ : $D \rightarrow N$ is normal if the family

$$
\mathscr{F}=\{f \circ g ; g \in \text { Aut } D\}
$$

is normal, where Aut $D$ denotes the holomorphic automorphism group of $D$.

Definition 2. We say that a subset $\mathscr{F}$ of $\operatorname{Hol}(D, N)$ is Aut $D$ invariant, if $f \circ g \in \mathscr{F}$ for every $f \in \mathscr{F}$ and every $g \in$ Aut $D$.

Received February 18, 1983. 
We study the condition of normality of holomorphic mappings.

Proposition 1. Let $d s_{D}^{2}$ be the Bergman metric of a homogeneous bounded domain $D$ in $C^{n}$ and $\left(N, d s_{N}^{2}\right)$ be a compact Hermitian manifold. If a holomorphic mapping $f: D \rightarrow N$ satisfies

$$
f^{*} d s_{N}^{2} \leqq C \cdot d s_{D}^{2}
$$

for a finite constant $C$, then $f$ is a normal holomorphic mapping.

Proof. As $d s_{D}^{2}$ is Aut $D$-invariant,

$$
(f \circ g)^{*} d s_{N}^{2}=g^{*} f^{*} d s_{N}^{2} \leqq C \cdot g^{*} d s_{D}^{2}=C \cdot d s_{D}^{2}
$$

for every $g \in$ Aut $D$. Hence $\mathscr{F}=\{f \circ g ; g \in$ Aut $D\}$ is equi-continuous. As $N$ is compact, $\mathscr{F} \subset \operatorname{Hol}(D, N)$ is normal according to Ascoli-Arzelà theorem.

q.e.d.

Theorem 1. Let $D$ be a bounded homogeneous domain in $C^{n}$ and $(N$, $\left.d s_{N}^{2}\right)$ be a Hermitian complex manifold. If a subset $\mathscr{F}$ of $\mathrm{Hol}(D, N)$ is an Aut $D$-invariant normal family, then there exists a constant $C$ such that $f^{*} d s_{N}^{2} \leqq C \cdot d s_{D}^{2}$ for every $f \in \mathscr{F}$.

Proof. Let

$$
C(z)=\sup _{\|x\|=1, f \in \mathscr{F}} f^{*} d s_{N}^{2} / d s_{D}^{2}(z, x)
$$

for each $z \in D$, and $x \in T_{z} C^{n}$, where \|\| is the length by a flat metric of $C^{n}$. We first prove $C(0)<\infty$ for a point $0 \in D$.

Suppose that $C(0)=\infty$. Then there exist sequences $\left\{f_{n}\right\}_{n=1}^{\infty} \subset \mathscr{F}$ and $\left\{x_{n} \in T_{0} C^{n},\left\|x_{n}\right\|=1\right\}$ of holomorphic tangent vectors such that

(i ) $f_{n}^{*} d s_{N}^{2}\left(0, x_{n}\right) \geqq n^{2} \cdot d s_{D}^{2}\left(0, x_{n}\right)$,

where we may assume that $\left\{x_{n}\right\}$ converges to $x \in T_{0} C^{n}$. From (i), we see

(ii) $\left\|f_{n *} x_{n}\right\|_{N} \geqq n \cdot \alpha$,

where \|\|$_{N}$ is the length measured by $d s_{N}^{2}$ and $\alpha$ is a positive constant. Since $\mathscr{F}$ is relatively compact in $\operatorname{Hol}(D, N)$, some subsequence $\left\{f_{n_{k}}\right\}_{k=1}^{\infty}$ of $\left\{f_{n}\right\}$ exists such that $f_{n_{k}} \rightarrow f \in \operatorname{Hol}(D, N)$ and $f_{n_{k}}(0) \rightarrow p \in N$ as $k \rightarrow \infty$. Consequently $f_{n_{k} *} x_{n_{k}} \rightarrow f_{*} x$ and $\left\|f_{n_{k} *} x_{n_{k}}\right\|_{N} \rightarrow\left\|f_{*} x\right\|_{N}<\infty$ as $k \rightarrow \infty$. This is contradictory to (ii) and hence $C(0)<\infty$.

We secondly prove that $C(z)$ is constant on $D$. From definition of $C(z)$

$$
f^{*} d s_{N}^{2}(z) \leqq C(z) \cdot d s_{D}^{2}(z)
$$

for every $f \in \mathscr{F}$. As $d s_{D}^{2}$ is Aut $D$-invariant, 


$$
(f \circ g)^{*} d s_{N}^{2}(z) \leqq C(g(z)) \cdot d s_{D}^{2}(z)
$$

for every $g \in$ Aut $D$. Hence $C(g(z)) \geqq C(z)$. Similarly $C(z) \geqq C(g(z))$ and so $C(z)=C(g(z))$. As $D$ is homogeneous, $C(z)$ is constant on $D$ and the theorem is proved.

q.e.d.

Corollary 1. Let $D$ and $N$ be as above. If $f: D \rightarrow N$ is a normal holomorphic mapping, then there exists a finite constant $C$ such that $f^{*} d s_{N}^{2}$ $\leqq C \cdot d s_{D}^{2}$.

\section{§2. Examples}

In this section, we give some examples of normal holomorphic mappings.

Example 1. Let $\Delta=\{|z|<1\} \subset C$ be the unit disc.

(i) We choose arbitrarily distinct $q$ points $p_{1}, \cdots, p_{q}$ in the Riemann sphere $\boldsymbol{P}^{1}$. Let $\mathscr{F}$ be a family of holomorphic mappings from $\Delta$ into $\boldsymbol{P}^{1}$ which satisfies the following condition (C).

(C) Every $f$ in $\mathscr{F}$ takes each value $p_{i} \in \boldsymbol{P}^{1}(i=1, \cdots, q)$ with multiplicity $\geqq m_{i}$ or $f$ omits a point $p_{i}$ and $\left\{m_{\imath}\right\}_{i=1}^{q}$ satisfies

$$
-2+\sum_{i=1}^{n}\left(1-\frac{1}{m_{i}}\right)>0
$$

where we set $m_{i}=\infty$ in the case $f$ omits $p_{i}$.

Then $\mathscr{F}$ is normal (Montel-Valiron, cf. Theorem 8.3 in H. Fujimoto [2]). Particularly, if a holomorphic map $f: \Delta \rightarrow P^{1}$ satisfies the condition (C), then $f$ is a normal holomorphic mapping, that is, a normal meromorphic function (cf. [7]).

(ii) Let $T=C / L$ be a complex torus and take a point $p$ of $T$. We consider the family $\mathscr{F}$ of all holomorphic mappings from $\Delta$ into $T$ which omit a value $p \in T$. Then $\mathscr{F}$ is normal. Particularly, every $f$ in $\mathscr{F}$ is a normal holomorphic mapping.

(iii) Let $V$ be a compact Riemann surface of genus $\geqq 2$. Then $\operatorname{Hol}(\Delta, V)$ is normal and hence every holomorphic mapping $f: \Delta \rightarrow V$ is normal.

(iv) Let $N$ be a paracompact connected complex analytic space and $M$ be a hyperbolically embedded subspace of $N$. Then $\operatorname{Hol}(D, M)$ is relatively compact in $\operatorname{Hol}(D, N)$ (See [5]). Particularly a holomorphic mapping from $D$ into $N$ is normal if the image of $f$ is contained in $M$. For such examples see M. L. Green [3] and [4]. 
Example 2. (i ) Let $d s_{\Delta}^{2}=d z d \bar{z} /\left(1-|z|^{2}\right)^{2}$ be a Bergman metric of the unit disc $\Delta$. We take homogeneous coordinates $\left(z_{0}, z_{1}\right)$ of $P^{1}$ and let $w=z_{1} / z_{0}$ for $z_{0} \neq 0$. Then Fubini-Study metric of $\boldsymbol{P}^{1}$ is given by

$$
d s_{P 1}^{2}=\frac{1}{\left(1+|w|^{2}\right)^{2}} d w d \bar{w}
$$

Consequently a meromorphic function $f: \Delta \rightarrow P^{1}$ is normal if and only if

$$
\frac{\left|f^{\prime}(z)\right|}{1+|f(z)|^{2}} \leqq C \cdot \frac{1}{1-|z|^{2}} \quad(z \in \Delta)
$$

for some finite constant $C$ (cf. [7]).

(ii) Let $T=C^{n} / L$ be an $n$-dimensional complex torus. Then $f: \Delta \rightarrow T$ is normal if and only if a lifting $\tilde{f}=\left(f_{1}, \cdots, f_{n}\right)$ of $f$ to $C^{n}$ satisfies

$$
\sum_{i=1}^{n}\left|f_{i}^{\prime}(z)\right|^{2} \leqq C \cdot \frac{1}{\left(1-|z|^{2}\right)^{2}}
$$

for some finite constant $C$. A holomorphic function satisfying condition (*) is classically called a Bloch function.

\section{$\S 3$. The estimate for characteristic functions}

In this section, we consider the case that $D$ is the unit ball $B^{n}=$ $\left\{\sum_{i=1}^{n}\left|z_{i}\right|^{2}<1\right\} \subset C^{n}$. The Bergman metric of $B^{n}$ is given by

$$
d s_{B^{n}}^{2}=\sum_{i, j=1}^{n} \frac{1}{\left(1-|z|^{2}\right)^{2}}\left[\left(1-|z|^{2}\right) \delta_{i j}+z_{i} \bar{z}_{j}\right] d z_{i} d \bar{z}_{j},
$$

where $|z|^{2}=\sum_{i=1}^{n}\left|z_{i}\right|^{2}$. Let $\left(N, d s_{N}^{2}\right)$ be a Hermitian manifold and let $\omega_{N}$, $\omega_{B^{n}}$ be the $(1,1)$-form associated with $d s_{N}^{2}, d s_{B^{n}}^{2}$ respectively. We calculate the characteristic function of a normal holomorphic mapping $f: B^{n} \rightarrow N$.

Lemma 1. Let $f: B^{n} \rightarrow N$ be a holomorphic mapping such that $f^{*} d s_{N}^{2}$ $\leqq C \cdot d s_{B^{n}}^{2}$, where $C$ is a constant. Set $\varphi=\sqrt{-1} / 2 \sum_{i=1}^{n} d z_{i} \wedge d \bar{z}_{i}$ and $B(r)$ $=\{|z|<r\}$. Then,

$$
\int_{B(r)} f^{*} \omega_{N} \wedge \varphi^{n-1} \leqq C \cdot \int_{B(r)} \omega_{B^{n}} \wedge \varphi^{n-1}
$$

Proof. We set

$$
\begin{aligned}
f^{*} \omega_{N} & =\frac{\sqrt{-1}}{2} \sum_{i, j=1}^{n} g_{i j} d z_{i} \wedge d \bar{z}_{j} \\
\omega_{B^{n}} & =\frac{\sqrt{-1}}{2} \sum_{i, j=1}^{n} h_{i \bar{j}} d z_{i} \wedge d \bar{z}_{j}
\end{aligned}
$$


Then,

$$
\begin{aligned}
f^{*} \omega_{N} \wedge \varphi^{n-1} & =(n-1) ! \cdot\left(\sum_{i=1}^{n} g_{i \bar{i}}\right) \prod_{j=1}^{n} \frac{\sqrt{-1}}{2} d z_{j} \wedge d \bar{z}_{j} \\
& =\frac{1}{n}\left(\sum_{i=1}^{n} g_{i \bar{i}}\right) \varphi^{n} .
\end{aligned}
$$

Since a matrix $\left(g_{i \bar{j}}\right)-C \cdot\left(h_{i \bar{j}}\right)$ is of negative semi-definite,

$$
g_{i \bar{i}} \leqq C \cdot h_{i \bar{i}} \quad(i=1, \cdots, n) .
$$

Hence,

$$
\begin{aligned}
\int_{B(\eta)} f^{*} \omega_{N} \wedge \varphi^{n-1} & =\frac{1}{n} \int_{B(r)}\left(\sum_{i=1}^{n} g_{i \bar{i}}\right) \varphi^{n} \leqq C \cdot \frac{1}{n} \int_{B(r)}\left(\sum_{i=1}^{n} h_{i \bar{i}}\right) \varphi^{n} \\
& =C \cdot \int_{B(r)} \omega_{L^{n}} \wedge \varphi^{n-1} .
\end{aligned}
$$

LEMMA 2.

$$
\begin{array}{r}
\frac{1}{\pi^{n}} \int_{0}^{r} \frac{d t}{t^{2 n-1}}\left(\int_{B(r)} \omega_{B_{n}} \wedge \varphi^{n-1}\right) \leqq \frac{1}{2} \log \frac{1}{1-r^{2}}+(n-1) \cdot C_{1} \\
(0<r<1),
\end{array}
$$

where $C_{1}=2 \log 2$.

Proof. Since the area of the boundary $\partial B(r)$ of $B(r)$ is $2 \pi^{n} /(n-1)$ !. $r^{2 n-1}$ and

$$
\begin{aligned}
& \omega_{B^{n}} \wedge \varphi^{n-1}=\frac{n !}{\left(1-|z|^{2}\right)^{2}}\left(1-\frac{n-1}{n}|z|^{2}\right) \cdot \frac{\varphi^{n}}{n !}, \\
& \begin{aligned}
\int_{B(t)} \omega_{B^{n}} \wedge \varphi^{n-1} & =\int_{0}^{t} \frac{n !}{\left(1-u^{2}\right)^{2}}\left(1-\frac{n-1}{n} u^{2}\right) \frac{2 \pi^{n}}{(n-1) !} u^{2 n-1} d u \\
& =2 \pi^{n} \int_{0}^{t} \frac{u^{2 n-1}}{\left(1-u^{2}\right)^{2}} d u+2 \pi^{n}(n-1) \int_{0}^{t} \frac{u^{2 n-1}}{1-u^{2}} d u .
\end{aligned}
\end{aligned}
$$

Using the estimates

$$
\int_{0}^{t} \frac{u^{2 n-1}}{\left(1-u^{2}\right)^{2}} d u \leqq t^{2 n-2} \int_{0}^{t} \frac{u d u}{\left(1-u^{2}\right)^{2}}
$$

and

$$
\int_{0}^{t} \frac{u^{2 n-1}}{1-u^{2}} d u \leqq t^{2 n-1} \int_{0}^{t}-\frac{d u}{1-u^{2}}
$$

we see 


$$
\int_{B(t)} \omega_{B^{n}} \wedge \varphi^{n-1} \leqq \pi^{n} \frac{t^{2 n}}{1-t^{2}}+(n-1) \pi^{n} \cdot t^{2 n-1}\left[\log \frac{1}{1-t}+\log (1+t)\right]
$$

Hence,

$$
\begin{aligned}
& \frac{1}{\pi^{n}} \int_{0}^{r} \frac{d t}{t^{2 n-1}}\left(\int_{B(t)} \omega_{B^{n}} \wedge \varphi^{n-1}\right) \\
& \quad \leqq \int_{0}^{r} \frac{t d t}{1-t^{2}}+(n-1) \int_{0}^{r}\left[\log \frac{1}{1-t}+\log (1+t)\right] d t \\
& \quad=\frac{1}{2} \log \frac{1}{1-r^{2}}+(n-1) C_{1}
\end{aligned}
$$

where

$$
C_{1}=\int_{0}^{1}\left[\log \frac{1}{1-t}+\log (1+t)\right] d t=2 \log 2 . \quad \text { q.e.d. }
$$

Definition 3. Let $B^{n},\left(N, d s_{N}^{2}\right)$ be as above and $f: B^{n} \rightarrow N$ be a holomorphic mapping. We define the characteristic function of $f$ by

$$
T(f, r)=\frac{1}{\pi^{n}} \int_{0}^{r} \frac{d t}{t^{2 n-1}}\left(\int_{B(t)} f^{*} \omega_{B^{n}} \wedge \varphi^{n-1}\right)
$$

Proposition 2. Let $\mathscr{F}$ be an Aut $B^{n}$-invariant normal family of holomorphic mapping from $B^{n}$ to $\left(N, d s_{N}^{2}\right)$. Then there exists a constant $C(r)$ depending only $r$ such that

$$
T(f, r) \leqq C(r) \quad(0 \leqq r<1)
$$

for every $f \in \mathscr{F}$.

Proof. Since there exists a constant $C$ such that $f^{*} d s_{N}^{2} \leqq C \cdot d s_{B^{n}}^{2}$ for every $f \in \mathscr{F}$, according to Theorem 1 , it is clear by Lemma 2 . We can take

$$
C(r)=\frac{C}{2} \log \frac{1}{1-r^{2}}+(n-1) C_{1} \cdot C . \quad \text { q.e.d. }
$$

Corollary. If a holomorphic mapping $f: B^{n} \rightarrow N$ is normal, then

$$
T(f \circ g, r)=O\left(\log \frac{1}{1-r}\right) \quad(r \longrightarrow 1)
$$

for every $g \in$ Aut $B^{n}$.

Remark 1. The property of the characteristic function for a normal holomorphic mapping is stronger than the property 


$$
T(f, r)=O\left(\log \frac{1}{1-r}\right) \quad(r \longrightarrow 1)
$$

Remark 2. If $N$ is the projective space $\boldsymbol{P}^{n}$, the inverse of the above proposition holds. This is clear by Theorem 5.10 in H. Fujimoto [2], because $B^{n}$ is a homogeneous domain.

\section{§4. A generalization of the big Picard theorem}

We set $\Delta=\{w \in C,|w|<1\}$ and $\Delta^{*}=\{w \in C, 0<|w|<1\}$. Let $\pi$ : $\Delta \rightarrow \Delta^{*}$ be the universal covering, where $\pi(w)=e^{(w+1) /(w-1)}$.

DeFinition 3. A holomorphic mapping $f$ from $\Delta^{*}$ into a complex analytic space $N$ is said to be normal if $f \circ \pi: \Delta \rightarrow N$ is a normal holomorphic mapping.

Theorem 2. Let $N$ be a paracompact complex manifold. If $f: \Delta^{*} \rightarrow N$ is a normal holomorphic mapping, then $f$ can be extended to a holomorphic mapping from $\Delta$ into $N$.

Proof. First of all, we show that there exists a sequence $\left\{z_{n}\right\}_{n=1}^{\infty} \subset \Delta^{*}$ converging to the origin 0 such that $\left\{f\left(z_{n}\right)\right\}_{n=1}^{\infty}$ converges to some point $p_{0}$ in $N$. Take a sequence $\left\{z_{n}\right\}_{n=1}^{\infty} \subset \Delta^{*}$ converging to 0 , points $\left\{w_{n}\right\}$ such that $\pi\left(w_{n}\right)=z_{n}$ and take $g_{n} \in$ Aut $\Delta$ such that $g_{n}(0)=w_{n}$. Consider the family $\mathscr{F}=\left\{f \circ \pi \circ g_{n}\right\}_{n=1}^{\infty}$. By the assumption, $\mathscr{F}$ is normal. We can take a subsequence $\left\{f \circ \pi \circ g_{n_{k}}\right\}_{n=1}^{\infty}$ of $\left\{f \circ \pi \circ g_{n}\right\}_{n=1}^{\infty}$ such that $\left\{f \circ \pi \circ g_{n_{k}}\right\}_{k=1}^{\infty}$ converges to some $h \in \operatorname{Hol}(\Delta, N)$. Particularly $f \circ \pi \circ g_{n_{k}}(0) \rightarrow h(0)=p_{0}$. We set $\pi \circ g_{n_{k}}(0)$ $=z_{k}$. Then $f\left(z_{k}\right) \rightarrow p_{0}$.

Secondary, we take a hermitian metric $d s_{N}^{2}$ of $N$. Since $f \circ \pi: \Delta \rightarrow N$ is normal, according to Theorem 1 of Section 1

$$
(f \circ \pi)^{*} d s_{N}^{2} \leqq C \cdot d s_{\lrcorner}^{2}
$$

where $C$ is a constant and $d s_{\Delta}^{2}=d z d \bar{z} /\left(1-|z|^{2}\right)^{2}$, (1) implies

$$
f^{*} d s_{N}^{2} \leqq C \cdot d s_{\Delta^{*}}^{2}
$$

where

$$
d s_{\Delta^{*}}^{2}=\frac{1}{4} \cdot \frac{d z d \bar{z}}{|z|^{2}(\log 1 / \mid z)^{2}} .
$$

We set $r_{k}=\left|z_{k}\right|$. It may be assumed that $\left\{r_{k}\right\}_{k=1}^{\infty}$ is a decreasing sequence. We denote by $d_{N}$ the distance of $N$ defined by $d s_{N}^{2}$. Then 


$$
d_{N}\left(f(z), p_{0}\right) \leqq d_{N}\left(f(z), f\left(z_{k}\right)\right)+d_{N}\left(f\left(z_{k}\right), p_{0}\right) .
$$

When $|z|=r_{k}$, according to (2)

$$
d_{N}\left(f(z), f\left(z_{k}\right)\right)=\frac{\sqrt{C}}{2} \int_{z_{k}}^{z} \frac{|d z|}{|d z| \log 1 /|z|} \leqq \sqrt{C} \cdot \frac{\pi}{\log 1 / r_{k}} .
$$

The right hand side converges to 0 as $k \rightarrow \infty$. According to (3) and (4), we see that, for every $\varepsilon$-neighbourhood $W$ of $p_{0}$, when $k_{0}$ is sufficiently large, $f\left(\left\{|z|=r_{k}\right\}\right)$ is contained in $W$ for $k \geqq k_{0}$. By the method in the proof of Theorem 3.1 in S. Kobayashi [6], Chapter VI, we can see that, for every neighbourhood $U$ of $p_{0}$, if $\delta>0$ is sufficiently small, $f\left(\left\{z \in \Delta^{*}\right.\right.$, $|z|<\delta\})$ is contained in $U$, and so we can define a holomorphic extension of $f$ to $\Delta$ by $f(0)=p_{0}$.

q.e.d.

Remark 3. The above theorem remains valid for a paracompact connected complex analytic space $N$, because $N$ has a hermitian metric $h$ in the extended sence, which induces the original topology of $N$, and the discussion of Section 1 and the above proof are available for such $(N, h)$ (see the introduction of P. Kiernan [5]).

Remark 4. Let $N$ be a paracompact connected complex analytic space and $M$ be hyperbolically embedded subspace of $N$. Then a holomorphic mapping $f: \Delta^{*} \rightarrow N$ with $f\left(\Delta^{*}\right) \subset M$ is normal. Hence, Theorem 2 is a generalization of a big Picard theorem given in S. Kobayashi [6], Chapter V, Theorem 6.1.

\section{$\S 5$. Asymptotic values}

In this section, we consider a generalization of the Lindelöf's theorem on asymptotic values of bounded holomorphic functions.

Definition 4. Let $f$ be a holomorphic mapping from a simply connected domain $G \subset C$ into a complex analytic space $N$. We define an angle with vertex at $z_{0} \in \partial G$ to be a domain $A$ defined as follows: Taking a boundary point $z_{1}\left(\neq z_{0}\right)$ of $G$ and a positive constant $\varepsilon$, we set

$$
A=\{z ; \varepsilon<\omega(z)<1-\varepsilon\},
$$

where $\omega(z)$ is the harmonic measure of the arc between $z_{0}$ and $z_{1}$ with respect to $G$.

We say that $f$ possesses the angular limit $p_{0}$ at $z_{0} \in \partial G$ if for every 
neighbourhood $U$ of $p_{0}$ and every angle $A$ with vertex at $z_{0}$, a neighbourhood $V$ of $z_{0}$ exists such that $f(V \cap A) \subset U$.

The following theorem is a generalization of Theorem 1 in the LehtoVirtanen [7].

THEOREM 3. Let $f$ be a holomorphic mapping from the unit disc $\Delta$ into a complex analytic space $N$. Suppose that $f$ has an asymptotic value $p_{0} \in N$ at a point $z_{0} \in \partial \Delta$ along a Jordan curve $\Gamma$ lying in $\Delta$ and that $f$ does not possess the angular limit $p_{0}$ at the point $z_{0}$. Then, there exists an arbitrarily small neighbourhood $U$ of $p_{0}$ such that, for every $\varepsilon>0$, there are a Jordan arc $L$ in $\Delta$ with end point at $z_{0}$ along which $f(z)$ tends to $p_{0}$ and a sequence $\left\{z_{n}\right\}_{n=1}^{\infty}$ in $\Delta$ converging to $z_{0}$ satisfying the condition $f\left(z_{n}\right) \in \partial U$ and $d_{\Delta}(z, L)<\varepsilon$, where $d_{\Delta}$ is the hyperbolic distance of $\Delta$.

For the proof, we need the following

LEMma (An extension of the Lindelöf's theorem). Let $r_{0}$ be greater than or equal to 0 and $D=\left\{z ;|z|>r_{0}, 0<\arg z<\alpha\right\}$. Let $f(z)$ be a bounded holomorphic function on $D$ which is continuous on $D \cup\left\{r \in R, r>r_{0}\right\}$ and $\limsup _{r \rightarrow \infty}|f(r)|=0$. Then for every $\delta>0$,

$$
\limsup _{\rho \rightarrow \infty}\{|f(z)| ; 0 \leqq \arg z \leqq \alpha-\delta,|z|>\rho\}=0 .
$$

Proof. This proof is suggested by H. Fujimoto.

We use the theory of harmonic measure (cf. [8], III, §2). We may take $\alpha=\pi$. As the case $r_{0}=0$ is well known, we may take $r_{0}$ is greater than 0 . We set $\delta^{\prime}=\delta / \pi$.

Let $\tilde{\omega}(z)$ be a harmonic measure of $\left\{|z|=r_{0}, \operatorname{Im} z>0\right\}$ with respect to $D=\left\{z ; \operatorname{Im} z>0,|z|>r_{0}\right\}$. Then, by the well-known theorem on solutions of Dirichlet problem (cf. [8], II, $\S 1$, p. 22-23) there exists $r_{1}>0$ such that

$$
\mid \tilde{\omega}(z)<\frac{\delta^{\prime}}{4} \quad \text { on } D^{\prime}=\left\{z ; z \in D,|z| \geqq r_{1}\right\} \text {. }
$$

We define harmonic functions $\omega_{1}, \omega_{2}$ for all $r_{2}$ greater than $r_{1}, r_{0}$ as follows.

$\omega_{1}(z)$ : harmonic measure of $\left\{z\right.$; real, $\left.z>r_{2}\right\}$ with respect to $D$,

$\omega_{2}(z)$ : harmonic measure of $\left\{z\right.$; real, $\left.z>r_{2}\right\}$ with respect to $\{\operatorname{Im} z>0\}$.

We see $\omega_{2}(z)=1-\arg \left(z-r_{2}\right) / \pi$. Then,

$$
\left\{\omega_{2}(z)>\frac{\delta^{\prime}}{2},|z|>r_{2}\right\} \supset\left\{\pi-\delta^{\prime} \cdot \pi>\arg z>0,|z|>r_{2}\right\}
$$


Also we see $\omega_{2}(z) \leqq \omega_{1}(z)+\tilde{\omega}(z)$ on $D$ by the maximum principle. As $\omega_{1}(z)$ $\geqq \omega_{2}(z)-\tilde{\omega}(z)$.

$$
\left\{\omega_{2}(z)>\frac{\delta^{\prime}}{2},|z|>r_{2}\right\} \subset\left\{\omega_{1}(z)>\frac{\delta^{\prime}}{4}\right\} \quad \text { on } D^{\prime} .
$$

Hence,

$$
\left\{\pi-\delta>\arg z>0,|z|>r_{2}\right\} \subset\left\{\omega_{1}(z)>\frac{\delta^{\prime}}{4}\right\} \quad \text { on } D^{\prime} .
$$

We define $m\left(r_{2}\right), m^{*}\left(r_{2}\right)$ as follows.

$$
\begin{aligned}
& m\left(r_{2}\right)=\sup \left\{|f(r)| ; r \text { is real, } r \geqq r_{2}\right\}, \\
& m^{*}\left(r_{2}\right)=\sup \left\{|f(z)| ; \pi-\delta>\arg z \geqq 0,|z| \geqq r_{2}\right\} .
\end{aligned}
$$

If $|f(z)| \leqq M$, then by two-constants theorem (cf. [8], III, §2, p. 41-42)

$$
m^{*}\left(r_{2}\right) \leqq m\left(r_{2}\right)^{\delta^{\prime} / 4} \cdot M^{1-\delta^{\prime} / 4}
$$

When $r_{2} \rightarrow \infty$, from assumption $m\left(r_{2}\right) \rightarrow 0$, and then $m^{*}\left(r_{2}\right) \rightarrow 0$. q.e.d.

Proof of Theorem 3. (This is essentially due to Lehto-Virtanen [7].)

We may suppose that $G=\{z ; 0<\arg z<\pi / 2\}$ and $z_{0}=\infty$, because the hyperbolic distance is conformally invariant. We may suppose $\Gamma$ starts at $z=0$ so that $\Gamma$ divides $G$ into two distinct parts $G_{1}$ and $G_{2}$. Let $G_{1}$ denote the part of $G$ bounded by $\Gamma$ and the imaginary axis. By assumption, $f$ does not converge uniformly to $p_{0}$ on some angle $A: \delta<\arg z<\pi / 2-2 \delta$ $(\delta>0)$. The same is also true at least in one of the intersections $G_{1} \cap A$ and $G_{2} \cap A$. We assume in the following that it is true in $G_{1} \cap A$. Let $U$ be a sufficiently small neighbourhood of $p_{0}$ which is biholomorphic to a subvariety $V$ in a bounded domain $W$ in $C^{m}$ by a map $\varphi$ and let $p_{0}$ be mapped to the origin 0 in $\boldsymbol{C}^{m}$.

We map $G_{1}$ onto the right angle $0<\arg w<\pi / 2$ and $\Gamma$ onto positive real axis $R^{+}$, keeping $0, \infty$ fixed. The image of $G_{1} \cap A$ lies in the angle $0<\arg w<\pi / 2-2 \delta$, as follows immediately if we apply the maximum principle to the harmonic measures of the real axis and $\Gamma$, respectively with respect to $G$ and $G_{1}$.

Consider $f^{-1}(U)$ and the connected component $U^{\prime}$ of $f^{-1}(U)$ which is a neighbourhood of $\Gamma \cap\{\infty>|z|>R\}$ for a large $R$ and let $U^{\prime \prime}$ be the image of $G_{1} \cap U^{\prime}$. The map $\varphi \circ f$ can be represented as $\varphi \circ f(w)=\left(f_{1}(w), \cdots\right.$, $\left.f_{m}(w)\right)$ on $f^{-1}(U)$, and each $f_{i}(w)(i=1, \cdots, m)$ satisfy $\left|f_{i}(w)\right|<M$, where $M$ is a constant. 
For some $U$, it is not possible that the upper part of the boundary of $U^{\prime \prime} \cap\{|z|>R\}$ is always above the line $\{\arg w=\pi / 2-\delta,|w|>R\}$ for some $R>0$, because otherwise $G_{1} \cap A \cap\{|z|>R\}$ is contained in $f^{-1}(U)$ for large $R$ and $f$ has uniform limit $p_{0}$ in $G_{1} \cap A$ according to the above lemma and it is a contradiction.

We can introduce a countable family of similar triangles $\left\{T_{\lambda}\right\}_{\lambda \in \Lambda}$ defined as follow: the base of $T_{\lambda}$ lies on the positive real axis, the two other sides are equal and the vertex angle is $\delta / 2$, for infinite $\Lambda^{\prime} \subset \Lambda$, the vertex of $T_{\lambda}\left(\lambda \in \Lambda^{\prime}\right)$ is in $\{0<\arg w<\pi / 2-\delta\} \cap \partial U^{\prime \prime}$ and $\bigcup_{\lambda \in \Lambda} T_{\lambda}$ is a simply connected domain which is contained in $U^{\prime \prime}$. We set $D=\bigcup_{\lambda \in \Lambda} T_{\lambda}$.

Let $\omega(w, D)$ be a harmonic measure with respect to $D$ which is equal to 0 on the real axis and is equal to 1 on the rest of the boundary. Since $f(z)$ tends to the limit $p_{0}$ along $\Gamma, f_{2}(w)$ tends to 0 along the positive real axis.

We consider the level line $L: \omega(w, D)=\lambda(0<\lambda<1)$. According to the above lemma, $f_{i}(w)$ tends to 0 along $L$, that is $f(w)$ tends to $p_{0}$ along $L$ as $w \rightarrow \infty$. By the above construction, we can take a sequence $\left\{\boldsymbol{z}_{n}\right\}_{n=1}^{\infty}$ $\subset \partial D$ and a triangle $T_{n}$ in $\left\{T_{\lambda}\right\}$ with vertex $z_{n}$ such that $f\left(z_{n}\right) \in \partial U$. Let $\omega\left(w, T_{n}\right)$ be a harmonic measure with respect to $T_{n}$ which is equal to 0 on the real axis and equal to 1 on the rest of the boundary. Since $T_{n}$ is contained in $D, \omega(w, D)<\omega\left(w, T_{n}\right)$ on $T_{n}$ by the maximum principle of harmonic measure. This implies that the level line $\omega(w, D)=\lambda$ is above the level line $\omega\left(w, T_{n}\right)=\lambda$ on $T_{n}$. We set $z_{n}=u+i \cdot v$. Let $Q_{1}\left(u+i \cdot v_{1}\right)$ and $Q_{2}\left(u+i \cdot v_{2}\right)$ be the intersections of the straight line $R e w=u$ and the level curves $\omega(w, D)=\lambda, \omega\left(w, T_{n}\right)=\lambda$, respectively. Then $v_{2}<v_{1}$. Since $G_{1} \subset G$, we see $d_{G}<d_{G_{1}}$, where $d_{G}, d_{G_{1}}$ are hyperbolic distance of $G, G_{1}$ respectively, and

$$
\begin{aligned}
d_{G}\left(z_{n}, Q_{1}\right)<d_{G_{1}}\left(z_{n}, Q_{1}\right) & \\
= & \int_{Q_{1}}^{z_{n}} \frac{1}{2} \cdot \sqrt{\frac{u^{2}+v^{2}}{u^{2} v^{2}}}|d w|<\frac{1}{2} \cdot \frac{1}{\sin \delta} \int_{Q_{1}}^{z_{n}} \frac{|d v|}{|v|} \\
\quad= & \frac{1}{2} \cdot \frac{1}{\sin \delta} \log \frac{v}{v_{1}}<\frac{1}{2} \cdot \frac{1}{\sin \delta} \log \frac{v}{v_{2}},
\end{aligned}
$$

where $v / v_{2}=k(\lambda)$ is independent on $z_{n}$. Since $k(\lambda) \rightarrow 1$ as $\lambda \rightarrow 1,\left\{z_{n}\right\}, L$ can be chosen such that

$$
d_{\Delta}\left(z_{n}, L\right)<\varepsilon \quad \text { for any } \varepsilon>0 .
$$

q.e.d.

We give a generalization of a result in Lehto-Virtanen [7]. 
TheOREM 4. Let $f: \Delta \rightarrow N$ be a normal holomorphic mapping and let $f$ have an asymptotic value $p_{0} \in N$ at a point $z_{0} \in \partial \Delta$ along a Jordan curve $J$ lying in 4 . Then $f$ possesses the angular limit $p_{0}$ at the point $z_{0}$.

Proof. We repeat the discussion of [7], p. 52 or [9], p. 86-87 for selfcontainedness of this paper.

Suppose that $f$ does not possess the angular limit $p_{0}$ at $z_{0}$. Then, by Theorem 3 , for a small neighbourhood $U$ of $p_{0}$ there exists a Jordan curve $L$ in $\Delta$ with end point at $z_{0}$ on which $f(z)$ tends to $p_{0}$, and a sequence $\left\{z_{n}\right\}_{n=1}^{\infty}$ which converges to $z_{0}$ and $f\left(z_{n}\right) \in \partial U$ and $d_{\Delta}\left(z_{n}, L\right)<M<\infty$. By compactness of $\partial U$, we can take $\left\{z_{n}\right\}_{n=1}^{\infty}$ such that $f\left(z_{n}\right) \rightarrow p^{\prime} \in \partial U$. Denote by $z^{\prime}=S_{n}(z)$ a function which maps $U$ conformally onto itself and satisfies the condition $S_{n}(0)=z_{n}$. We denote by $K$ the hyperbolic circle with center at $z=0$ and hyperbolic radius $M+1$. Since $d_{\Delta}$ is invariant by $S_{n}, S_{n}^{-1}$ maps some arcs of $L$ into $K$. We denote by $C_{n}$ the unions of such arcs of $L$ for a number $n$. Since $\left\{f \circ S_{n}\right\}$ is a normal family, a subsequence $\left\{f \circ S_{n_{k}}\right\}_{k=1}^{\infty}$ converges to a holomorphic mapping $\varphi: \Delta \rightarrow N$. As $f\left(S_{n}(0)\right)=$ $f\left(z_{n}\right)=p_{n}$ converges to $p^{\prime}\left(\neq p_{0}\right), \varphi(0)=p^{\prime}$. While, $C=\bigcap_{m \geqq 1} \overline{\bigcup_{n \geqq m} S_{n}^{-1}\left(C_{n}\right)}$ $\subset K$ possesses a continuum on which $\varphi(z)=p_{0}$, and hence $\varphi(z) \equiv p_{0}$. We have thus arrived at a contradiction and the theorem is proved. q.e.d.

Corollary. Let $N$ be a paracompact connected complex analytic space and let $M$ be a hyperbolically embedded subspace of $N$. We consider a holomorphic mapping $f: \Delta \rightarrow N$ with $f(\Delta) \subset M$. If $f$ has an asymptotic value $p_{0} \in N$ at $z_{0} \in \partial \Delta$ along a Jordan curve $J$ lying in $\Delta$, then $f$ possesses the angular limit $p_{0}$ at $z_{0}$.

Therefore we can see that the Lindelöf's theorem holds for the following mappings (cf. §2, Example 1, (iv)).

Example 3. (i) Let $f: \Delta \rightarrow \boldsymbol{P}^{n}$ be a holomorphic mapping which omits $D=\bigcup_{i=1}^{2 n+1} H_{i}$, where $\left\{H_{i}\right\}_{i=1}^{2 n+1}$ are hyperplanes in general position.

(ii) Let $f: \Delta \rightarrow T=C^{n} / L$ be a holomorphic mapping which omits $D$ $\subset T$, where $D$ is a hypersurface of $T$ not containing a proper complex subtorus.

We give an interesting example of the non-normal holomorphic mapping.

Example 4. We denote $\left(w_{0}, w_{1}, w_{2}\right)$ the homogeneous coordinates of $\boldsymbol{P}^{2}$. We define a holomorphic mapping $f: \Delta \rightarrow \boldsymbol{P}^{2}$ by 


$$
z \longmapsto\left(2, z+1,(1-z) e^{(1+z) /(1-z)}\right)
$$

and four complex lines $\ell_{0}, \ell_{1}, \ell_{2}, \ell_{3}$ in $P^{2}$ in general position as follows,

$$
\begin{array}{ll}
\ell_{0}: w_{0}=0, & \ell_{1}: w_{1}=0, \\
\ell_{2}: w_{1}-w_{2}=0, & \ell_{3}: w_{0}-w_{1}-w_{2}=0 .
\end{array}
$$

Then we can easily see that $f$ omits $D=\bigcup_{i=0}^{3} \ell_{i}$. We remark that $\operatorname{Re}(1+z) /(1-z)=1$ on $\{z \in \Delta ;|z-1 / 2|=1 / 2\}$ and $\lim _{r \rightarrow 1-0}(1-r) e^{(1+r) /(1-r)}$ $=\infty$. Hence, we see that $f(z)$ has an asymptotic value $(1,1,0) \in \boldsymbol{P}^{n}$ at $z=1$ along a curve $\{z \in \Delta,|z-1 / 2|=1 / 2\}$, while $f$ has a radial limit $(0,0,1)$ $\in P^{2}$ at $z=1$. Therefore $f$ is not a normal holomorphic mapping according to Theorem 4. But this mapping $f$ is of bounded type (i.e. $\limsup _{r \rightarrow 1} T(f, r)$ $<\infty)$.

In fact, let $f^{*} d s_{P_{2}}^{2}=\rho(f(z))^{2} d z d \bar{z}$, where $d s_{P_{2}}^{2}$ is the Fubini-Study metric on $\boldsymbol{P}^{2}$, then $\rho(f(z))=\partial^{2} / \partial z \partial \bar{z} \log \|f(z)\|^{2}$, where

$$
\|f(z)\|^{2}=\sum_{i=0}^{2}\left|f_{i}\right|^{2}, \quad f=\left(f_{0}, f_{1}, f_{2}\right),
$$

and we have

$$
\begin{aligned}
T(f, r) & =\frac{1}{\pi} \int_{0}^{r} \frac{d t}{t} \int_{\Delta(t)} \rho(f(z))^{2} d x d y \\
& =\frac{1}{2 \pi r} \int_{0}^{2 \pi} \log \left\|f\left(r e^{i \theta}\right)\right\| d \theta-\log \|f(0)\|
\end{aligned}
$$

Using

$$
\frac{1}{2 \pi} \int_{0}^{2 \pi} \frac{1-r^{2}}{\left|1-r e^{i \theta}\right|^{2}} d \theta=1 \quad(0 \leqq r<1),
$$

we easily see $T(f, r) \leqq C$, where $C$ is some finite constant.

§6. Hyperbolic analysis and generalization of the Lindelöf's theorem

Let $M$ be a connected complex analytic space, $d_{M}$ be its Kobayashi pseudo-distance (cf. [6]), and let $A(M)$ be the set of non-hyperbolic points, i.e.

$$
\begin{aligned}
& A(M)=\{p \in M ; \text { there exists a point } q \in M \text { such that } \\
& \left.\qquad q \neq p \text { and } d_{M}(p, q)=0\right\} .
\end{aligned}
$$

We remark that $d_{M}(p, q)$ is continuous with respect to $p$ and $q$ (cf. T. J. Barth [1]). 
We give a condition of normality for holomorphic mapping in the term of hyperbolic analysis.

Proposition 3. Let $M$ be a connected compact complex manifold and let $f: \Delta \rightarrow M$ be a holomorphic mapping. We define a global cluster set of $f$ by

$$
\begin{aligned}
& C(f)=\left\{p \in M \text {; there exists a sequence }\left\{z_{n}\right\}_{n=1}^{\infty} \subset \Delta\right. \text { such that } \\
& \left.\left|z_{n}\right| \longrightarrow 1, f\left(z_{n}\right) \longrightarrow p\right\} \text {. }
\end{aligned}
$$

If $C(f) \cap A(M)=\phi$, then $f$ is a normal holomorphic mapping.

Proof. Suppose that $f$ is not normal. We take a hermitian metric $d s_{M}^{2}$ of $M$. Then there exists some sequence $\left\{G_{n}\right\}_{n=1}^{\infty} \subset$ Aut $\Delta$ such that

$$
\left(f \circ G_{n}\right) * d s_{M}^{2} / d s_{\Delta}^{2}\left(0, \frac{\partial}{\partial z}\right) \longrightarrow \infty \quad(n \longrightarrow \infty)
$$

and $\left|G_{n}(0)\right| \rightarrow 1(n \rightarrow \infty)$ according to Section 1 .

We set $g_{n}=f \circ G_{n}$ and let $p \in M$ be an accumulated point of the set $\left\{g_{n}(0)\right\}_{n=1}^{\infty} \subset M$. (1) implies

$$
\left\|g_{n^{*}}\left(\frac{\partial}{\partial z}\right)_{z=0}\right\|_{M} \longrightarrow \infty
$$

where \|\|$_{M}$ is the length measured by $d s_{M}^{2}$. Let $U$ be a sufficiently small coordinate neighbourhood of $p$. Using the Cauchy estimate, we see that for any positive integer $n$ and sufficiently large $m$

$$
g_{n}\left(\Delta\left(\frac{1}{m}\right)\right) \cap \partial U \neq \phi
$$

where $\Delta(1 / \mathrm{m})$ is a disc of radius $1 / \mathrm{m}$. That is, there exists a sequence $\left\{x_{m}\right\}_{m=1}^{\infty} \subset \partial U$ such that $x_{m} \in g_{n}(\Delta(1 / m))$. We see

$$
d_{M}\left(g_{n}(0), x_{m}\right) \leqq d_{\Delta}\left(0, \frac{1}{m}\right) \longrightarrow 0 \quad(m \longrightarrow \infty)
$$

By continuity of $d_{M}(p, q)$, we see that there exists a point $q \in \partial U$ such that $d_{M}(p, q)=0$, that is $p \in A(M)$. This is a contradiction and the theorem is proved.

q.e.d.

We give another type of generalizations of the Lindelöf's theorem.

THEOREM 5. Let $f$ be a holomorphic mapping from $\Delta$ to a connected 
complex analytic space $M$. Suppose that $f$ has an asymptotic value $p_{0} \in M$ at $z_{0} \in \partial \Delta$ along a Jordan curve in $\Delta$ and that $f$ does not possess the angular limit $p_{0}$ at $z_{0}$. Then $p_{0} \in \overline{A(M)}$.

Proof. We remark that $d_{M}$ induces the usual topology on $M-\overline{A(M)}$ (cf. Barth [1]). Suppose $p_{0}$ is not in $\left.\overline{A(M}\right)$. Then we can take 2 $\varepsilon$-neighbourhood $U$ with respect to $d_{M}$ which is contained in $M-\overline{A(M)}$. According to Theorem 3 , there exist a Jordan curve $L$ in $\Delta$ with end point $z_{0}$ and a sequence $\left\{z_{n}\right\}_{n=1}^{\infty}$ which converges to $z_{0}$ such that $f$ has an asymptotic value $p_{0}$ along $L$ and satisfy the condition $f\left(z_{n}\right) \in \partial U$ and $d_{4}\left(z_{n}, L\right)<\varepsilon$.

Let $u_{n}$ be points of $L$ which satisfy $d_{\Delta}\left(z_{n}, u_{n}\right)<\varepsilon$. Then,

$$
d_{M}\left(f\left(z_{n}\right), p_{0}\right) \leqq d_{M}\left(f\left(z_{n}\right), f\left(u_{n}\right)\right)+d_{M}\left(f\left(u_{n}\right), p_{0}\right) .
$$

For large $n$,

$$
d_{M}\left(f\left(u_{n}\right), p_{0}\right)>\frac{1}{2} \varepsilon
$$

On the other hand,

$$
d_{M}\left(f\left(z_{n}\right), f\left(u_{n}\right)\right) \leqq d_{\lrcorner}\left(z_{n}, u_{n}\right)
$$

Hence,

$$
d_{M}\left(f\left(z_{n}\right), p_{0}\right)<\frac{3}{2} \varepsilon \quad \text { for large } n
$$

As $d_{M}\left(f\left(z_{n}\right), p_{0}\right)=2 \varepsilon$, this is a contradiction and hence the theorem is proved.

q.e.d.

Corollary. Let $M$ be a hyperbolic complex space and $f: \Delta \rightarrow M$ be $a$ holomorphic mapping. If $f$ has an asymptotic value $p_{0} \in M$ at a point $z_{0}$ $\in \partial \Delta$ along a Jordan curve lying in $\Delta$, then $f$ possesses the angular limit $p_{0}$ at $z_{0}$.

\section{REFERENCES}

[1] T. J. Barth, The Kobayashi distance induces the standard topology, Proc. Amer. Math. Soc., 35 (1972), 439-441.

[2] H. Fujimoto, On families of meromorphic maps into the complex projective space, Nagoya Math. J., 54 (1974), 21-51.

[3] M. L. Green, The hyperbolicity of the complement of $2 n+1$ hyperplanes in general position in $\boldsymbol{P}_{n}$ and related result, Proc. Amer. Math. Soc., 16 (1977), 109-113.

[4] M. L. Green, Holomorphic maps to complex tori, Amer. J. Math., 100 (1977), $615-620$. 
[5] P. Kiernan, Hyperbolically imbedded space and the big Picard theorem, Math. Ann., 204 (1973), 203-209.

[6] S. Kobayashi, Hyperbolic manifolds and holomorphic mappings, Marcel Dekker, Inc., New York, 1970.

[ 7 ] O. Lehto and K. I. Virtanen, Boundary behaviour and normal meromorphic functions, Acta Math., 97 (1957), 47-65.

[ 8 ] R. Nevanlinna, Analytic functions, Springer-Verlag, New York-Heidelberg-Berlin, 1970.

[9] K. Noshiro, Cluster sets, Ergebniss der Math., Heft 28, Springer-Verlag, BerlinGöttingen-Heidelberg, 1960.

Production Technology Laboratories

Engineering Center

Sharp Corpolation

2613-1 Ichinomoto-cho, Tenri-shi, Nara 632

Japan 